\title{
Infectious complications during connective tissue diseases: A prospective study on 234 cases
}

\section{Boubacar Ahy Diatta, Douja Hamdi, Ndeye Bougoul Seck, Saer Diadie, Aminata Deh, Khadim Diop, Mame Tening Ndiaye, Assane Diop, Maodo Ndiaye, Moussa Diallo, Suzanne Oumou Niang}

Department of Dermatology of the Cheikh Anta Diop University of Dakar, Avenue Pasteur BP: 16520 Dakar, Senegal

Corresponding author: Dr. Boubacar Ahy Diatta, E-mail: ahydiatta@yahoo.com

\begin{abstract}
Background: The infectious complications during connective tissue diseases are favored by immunosuppression linked on the one hand to systemic disease and on the other hand to their treatment. The aim of this study was to identify the different infectious complications associated with these systemic diseases. Materials and Methods: It was a multicenter descriptive study over a period of 8 months in two Dermatology departments in Dakar. All patients followed for connective tissue disease who had evidence of infection during the study period were included. Results: We identified 231 cases of connective tissue disease. We had noted the occurrence of an infection in 56 cases. Infections were noted during: systemic scleroderma in 19 cases, systemic lupus in 15 cases, mixed connective tissue disease in 12 cases and dermatomyositis in 10 cases. The different types of infection that were observed during these systemic diseases were fungal (4lcas), bacterial (34cas), viral (12cas) and parasitic (6cas). Patients developed an infection before taking corticosteroids in 34 cases (36.6\%) and after taking corticosteroids in 59 cases (63.4\%). Conclusion: Connectivites are a group of autoimmune diseases that are exposed to an infectious risk during their evolution. The search for these infections at the beginning and during the treatment of these system diseases improves their prognosis.
\end{abstract}

Key words: Systemic diseases; Infection; Corticosteroids

How to cite this article: Diatta BA, Hamdi D, Seck NB, Diadie S, Deh A, Diop K, Ndiaye MT, Diop A, Ndiaye M, Diallo M, Niang SO. Infectious complications during connective tissue diseases: A prospective study on 234 cases. Our Dermatol Online. 2020;11(1):13-19.

Submission: 09.04.2019; Acceptance: 09.06.2019

DOI:10.7241/ourd.20201.3 


\title{
Les complications infectieuses au cours des connectivites: Une étude prospective sur 234 cas
}

\section{Boubacar Ahy Diatta, Douja Hamdi, Ndeye Bougoul Seck, Saer Diadie, Aminata Deh, Khadim Diop, Mame Tening Ndiaye, Assane Diop, Maodo Ndiaye, Moussa Diallo, Suzanne Oumou Niang}

Department of Dermatology of the Cheikh Anta Diop University of Dakar, Avenue Pasteur BP: 16520 Dakar, Senegal

Corresponding author: Dr. Boubacar Ahy Diatta, E-mail: ahydiatta@yahoo.com

\begin{abstract}
RESUME
Introduction: Les complications infectieuses au cours des connectivites sont favorisées par l'immunodépression liée d'une part à la maladie de système et d'autre part à leur traitement. Notre objectif était d'identifier les différentes complications infectieuses associées à ces connectivites. Matériels et méthodes: Il s'agissait d'une étude multicentrique descriptive sur une période de 8 mois dans deux services de Dermatologie à Dakar. Tous les malades suivis pour une connectivite et qui présentaient des signes d'infection durant la période de l'étude ont été inclus. Résultats: Nous avons recensé 231 cas de connectivite. Nous avions noté la survenue d'une infection dans 56cas soit une prévalence hospitalière des infections de 24,2\%. Les infections étaient notées au cours: de la sclérodermie systémique dans 19 cas, du lupus systémique dans $15 \mathrm{cas}$, de la connectivite mixte dans $12 \mathrm{cas}$ et de la dermatomyosite dans $10 \mathrm{cas}$. Les différents types d'infection qui ont été observés au cours de ces connectivites étaient d'origine fongique (4lcas), bactérienne (34cas), virale (12cas) et parasitaire (6cas). Les malades ont développé une infection avant la prise des corticoïdes dans 34cas (36,6\%) et après la prise des corticoïdes dans 59cas (63,4\%). Conclusion: Les connectivites constituent un groupe de maladies auto-immunes qui sont exposés à un risque infectieux au cours de leur évolution. La recherche de ces infections au début et au cours du traitement de ces maladies de système permet d'améliorer leurs pronostics.
\end{abstract}

Mots clés: Connectivite; Infection; Corticothérapie

\section{INTRODUCTION}

Les connectivites regroupent avec les vascularites les «maladies systémiques » qui sont des affections auto-immunes et/ou inflammatoires non spécifiques d'organe. Les manifestations dermatologiques constituent souvent une circonstance de découverte et facilitent le diagnostic précoce $[1,2]$. La gravité est liée au diagnostic tardif, aux atteintes viscérales notamment la néphropathie glomérulaire, la fibrose pulmonaire, les troubles du rythme et de la conduction cardiaque et le risque de sepsis lié aux complications infectieuses $[3,4]$. Ces complications infectieuses sont secondaires à l'immunodépression causée par la connectivite ainsi qu'aux effets iatrogènes du traitement par les corticoïdes et les immunosuppresseurs utilisés dans ces pathologies auto-immunes [5-8]. Lobjectif de cette étude était d'identifier d'une part les différentes complications infectieuses observées dans ces connectivites, d'autre part les facteurs de risque associés à ces infections ainsi que leurs modalités de prise en charge.

\section{MATÉRIELS ET MÉTHODES}

Il s'agissait d'une étude multicentrique descriptive avec un recrutement prospectif réalisée de janvier 2018 en Aout 2018 (8 mois) dans les deux services de Dermatologie à Dakar. Nous avons inclus tous les malades suivis en hospitalisation ou en externe pour une connectivite et qui présentaient des signes d'infection durant la période de l'étude. Le diagnostic de connectivites était retenu selon

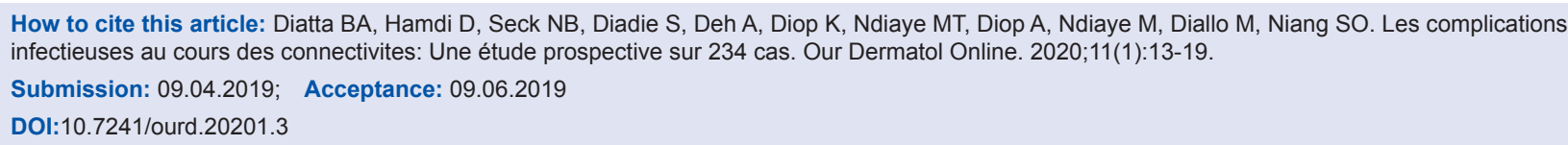


les manifestations dermatologiques de la connectivite et les critères de l'American College of Rheumatology ACR (1997) pour le lupus systémique, de l'EULAR (2013) pour la sclérodermie systémique et de Bohan et Peter pour la dermatomyosite.

Le diagnostic d'infection était retenu devant:

I. des signes cliniques

- généraux: température $<36^{\circ} \mathrm{C}$ ou $>38^{\circ} \mathrm{C}$; fréquence cardiaque $>90 / \mathrm{min}$; fréquence respiratoire $>20 / \mathrm{min}$

- dermatologiques: squames du cuir chevelu, lésions érythémato-squameuses prurigineuses, lésions vésiculo-bulleuses douloureuses, muguet buccal, croutes mélicèriques, ulcérations douloureuses

- Des signes extra-dermatologiques: brulures mictionnelles, toux, râles à l'auscultation pulmonaire, douleurs auriculaires, diarrhée.

II. des explorations paracliniques

- Biologiques à la recherche d'une: CRP élevée, hyperleucocytose ou leucopénie.

- Bactériologiques: hémocultures, examen cytobactériologique des urines ou des crachats

- Parasitaires: prélèvement vaginal, selles KAOP

- Mycologique: prélèvement mycologique, scotch test

- Radiologiques: radiographie de thorax, scanner thoracique, échographie abdominale et pelvienne, échographie des parties molles, radiographie panoramique dentaire.

Le traitement anti-infectieux était instauré selon le type d'infection.

Lévaluation était faite à J14 et J30 sur la base des données cliniques et paracliniques de contrôle.

La saisie et l'analyse des données étaient effectuées sur le logiciel SPSS IBM Statistics version 22. Létude descriptive était réalisée par le calcul de fréquences pour les variables qualitatives. Pour les données quantitatives, l'étude était réalisée par le calcul des moyennes.

Létude analytique était faite avec des croisements de variables à l'aide de tableaux de contingence à double entrée. Pour comparer les fréquences, le test du KHI 2 et celui de Fischer étaient utilisés, avec un seuil de significativité alpha inférieur à 0,05 .

\section{RÉSULTATS}

Nous avons recensé 231 cas de connectivite parmi 7685 malades suivis durant la période de l'étude soit une fréquence hospitalière de $3 \%$.
Parmi ces 231 cas de connectivite, nous avions noté la survenue d'une infection dans 56 cas. La prévalence hospitalière des infections au cours des connectivites était de 24,2\%. Les connectivites dont survenaient ces infections étaient par ordre de fréquence la sclérodermie systémique (88cas), le lupus systémique (70cas), la connectivite mixte (42cas) et la dermatomyosite (3lcas). Les connectivites mixtes associaient plusieurs maladies systémiques notamment (la sclérodermie systémique et la dermatomyosite) dans 5cas, (le lupus systémique et la dermatomyosite) dans 3cas, (le lupus systémique et la sclérodermie systémique) dans 2cas, (le lupus systémique, la sclérodermie systémique et la dermatomyosite) dans 2 cas. Les malades étaient de sexe féminin dans 46cas et de sexe masculin dans 10 cas soit un sexe ratio de 0.21 .

Lâge moyen était de 40,5 \pm 13,9 ans avec des extrêmes de 16 et 80 ans.

Les circonstances de découverte des infections étaient fortuites lors du suivi de la connectivite dans 22 cas $(39,3 \%)$, soit au décours d'une poussée de la connectivite dans 19 cas $(33,9 \%)$ ou avec des signes fonctionnels de l'infection dans 15 cas $(26,8 \%)$. La durée d'évolution des connectivites était inférieure à une année dans 22 cas $(39,3 \%)$, entre lan et 3 ans dans 21 cas $(37,5 \%)$, entre 3 ans et 5 ans dans 8 cas $(14,3 \%)$ et supérieur à 5 ans dans 5 cas $(8,9 \%)$.

Les infections étaient notées au cours: de la sclérodermie systémique dans 19cas, du lupus systémique dans 15 cas, de la connectivite mixte dans 12 cas et de la dermatomyosite dans 10 cas. Les différents types d'infection qui ont été observés au cours de ces connectivites étaient d'origine fongique (4lcas), bactérienne (34cas), virale (12cas) et parasitaire (6cas). Les malades ont développé une infection avant la prise des corticoïdes dans 34cas (36,6\%). Ces infections étaient d'origine fongique (15cas), bactérienne (12cas), virale (4cas) et parasitaire (3cas). La fig. 1 illustre la répartition des différents types d'infections survenues avant la prise des corticoïdes en fonction des connectivites. Les malades ont présenté une infection après la prise des corticoïdes dans 59cas $(63,4 \%)$.Ces infections étaient d'origine mycosique (27cas), bactérienne (2lcas), virale (8cas) et parasitaire (3cas). La fig. 2 illustre la répartition des différents types d'infections survenues après la prise des corticoïdes en fonction des connectivites. Les malades qui présentaient une infection étaient à des doses de corticoïdes variables. La répartition des malades en fonction de la dose de 
prise orale des corticoïdes est illustrée sur la fig. 3. Les corticoïdes étaient associés aux immunosuppresseurs dans 9cas parmi lesquels le méthotrexate (4cas), l'azathioprine (2cas) et le cyclophosphamide en bolus (3cas). Les infections avaient une durée d'évolution variable en fonction des connectivites. Cette durée était inférieure à une semaine dans 21 cas, entre 1 et 4 semaines dans 25 cas, supérieure à 4 semaines dans 10 cas. Les localisations des différentes infections sont illustrées dans le tableau 1. Les types d'infection étaient variables en fonction des différentes connectivites. Le tableau 2 illustre la répartition des malades selon le type d'infection. Les agents pathogènes ont été isolés dans 26cas (46,4\%). Il s'agissait d'agent pathogène bactérien: Bacille de Koch, Salmonella enterica,

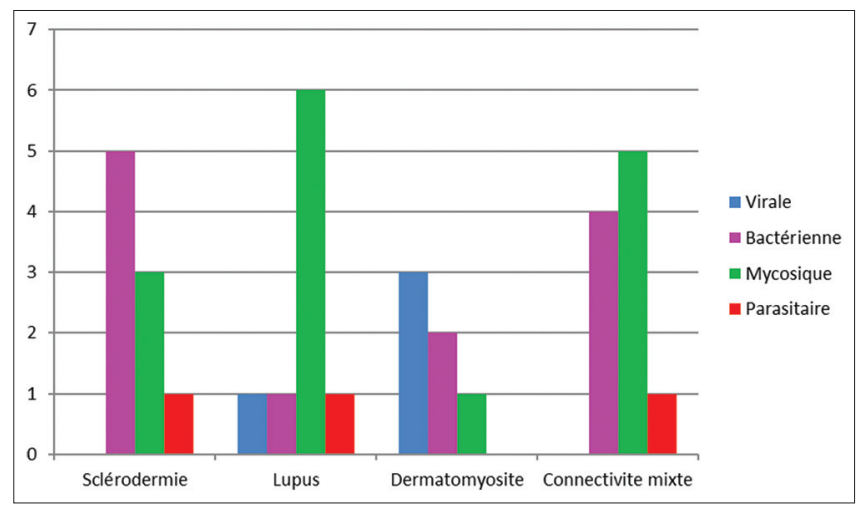

Figure 1: Répartition des types d'infection avant la prise de corticoïde.

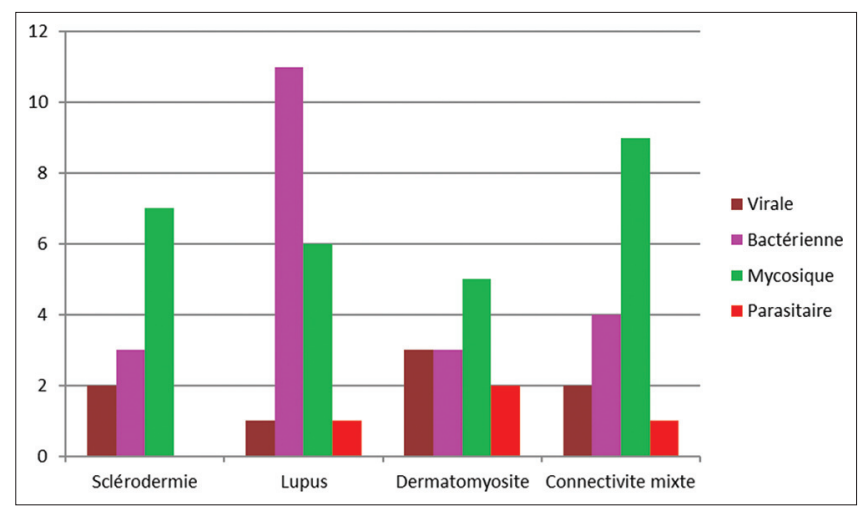

Figure 2: Répartition des types d'infection après la prise de corticoïde.
Escherichia coli, Proteus mirabilis, Staphylocoque aureus, Acinetobacter, Klebsiella pneumoniae; fongiques: Microsporum canis, Trichophyton rubrum, Candida albicans, Trichophyton mentagraphytes, Trichophyton schoenleinii et parasitaires: Trichomonas vaginalis. Les différents types de traitement local et général utilisés pour la prise en charge des infections sont répertoriés sur le tableau 3. Lévolution des infections était marquée par une guérison dans 7 cas au cours du lupus systémique, dans 17 cas dans la sclérodermie systémique, 5 cas dans la dermatomyosite et 9 cas au cours des connectivites mixtes. A l'étude analytique les connectivites mixtes étaient plus associées aux épisodes infectieux itératives $(p=0.009)$. La dermatomyosite était statistiquement plus liée à la survenue d'une infection virale $(\mathrm{p}=0.018)$ et la sclérodermie plus aux infections bactériennes $(\mathrm{p}=0.048)$.La prise de corticoïdes était associée aux risque de survenue des infections $(\mathrm{p}<0.001)$.

\section{DISCUSSION}

Notre rapportons une fréquence élevée de 24,2\% des infections au cours des connectivites. Les circonstances de découverte étaient le plus souvent fortuites dans $39,3 \%$. Ces infections survenaient le plus souvent après l'administration des corticoïdes dans $63,4 \%$ et parfois existaient avant la corticothérapie dans 36,6\%. Elles étaient à prédominance mycosiques et bactériennes avec une fréquence particulière des infections virales au cours de la dermatomyosite et des infections bactériennes au cours de la sclérodermie. La fréquence des infections $(21,4 \%)$ au cours du lupus systémique dans notre étude est comparable aux autres études rapportées en Inde (26,5\%) et au Canada (25\%) [9,10]. Cependant la fréquence de ces infections était plus élevée en France (40\%) [3]. Concernant la fréquence des infections au cours de la sclérodermie systémique, très peu d'études s'y sont intéressés et elles portaient seulement sur les infections opportunistes, ces dernières représentent 2 à $9 \%$ des causes de décès au cours de cette maladie [11].

Table 1: Répartition des malades selon la localisation de l'infection

\begin{tabular}{|c|c|c|c|c|c|}
\hline Localisations & Lupus & Sclérodermie & Dermatomyosite & $\begin{array}{l}\text { Connectivites } \\
\text { mixtes }\end{array}$ & Total \\
\hline Peau & 14 & 19 & 16 & 19 & 68 \\
\hline Poumon & 3 & 3 & 1 & 0 & 7 \\
\hline Urogénitale & 0 & 2 & 1 & 4 & 7 \\
\hline Digestive & 0 & 4 & 0 & 1 & 5 \\
\hline ORL & 2 & 1 & 0 & 1 & 4 \\
\hline Ganglionnaire & 1 & 0 & 0 & 0 & 1 \\
\hline Musculaire & 0 & 0 & 1 & 0 & 1 \\
\hline Total & 20 & 29 & 19 & 25 & 93 \\
\hline
\end{tabular}


Dans notre étude, 32,1\% de cas de lupus avaient développé des infections avant la prise des corticoïdes ce qui rejoint l'étude de Zonana qui ont rapporté une fréquence de $40 \%$ au cours du lupus systémique [12]. Cette immunodépression semble être liée d'une part à une hyperactivation spontanée des lymphocytes $\mathrm{B}$ à l'origine d'une augmentation de la production d'immunoglobulines et d'autre part à des anomalies quantitatives et qualitatives des lymphocytes T. La résultante est une baisse de la synthèse de divers facteurs (interleukines 1 et 2 , interférons, facteurs de croissance hématopoïétiques) qui joue un rôle majeur anti-infectieux [13-15].

Cependant dans notre étude, la majorité de nos patients $(67,9 \%)$ avaient développé des infections au cours du traitement par les corticoïdes avec un lien statistiquement significatif entre la survenue des infections et la prise de corticoïdes $(\mathrm{p}<0.001)$. Ceci rejoint les résultats d'une méta-analyse comparant les corticoïdes versus placebo qui montrait que la prise de corticoïdes était un facteur de risque d'infection avec un risque relatif de 1,6 [8]. Une étude en France

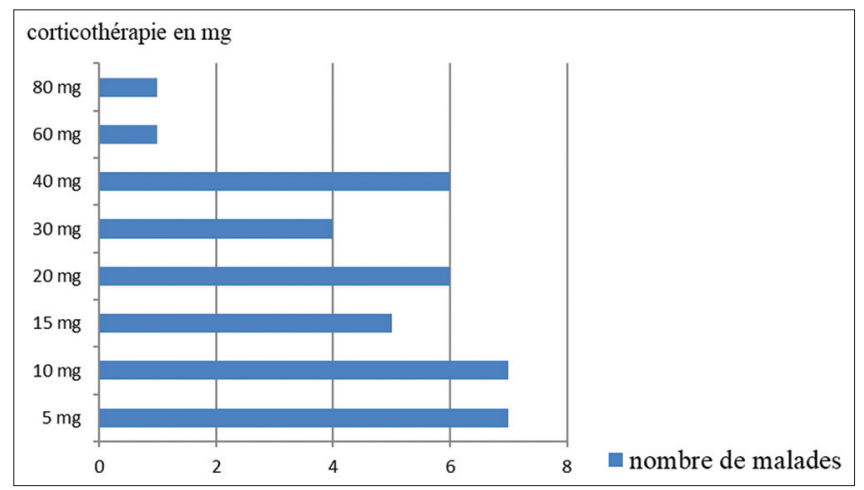

Figure 3: Répartition des malades selon la dose de prise des corticoïdes.

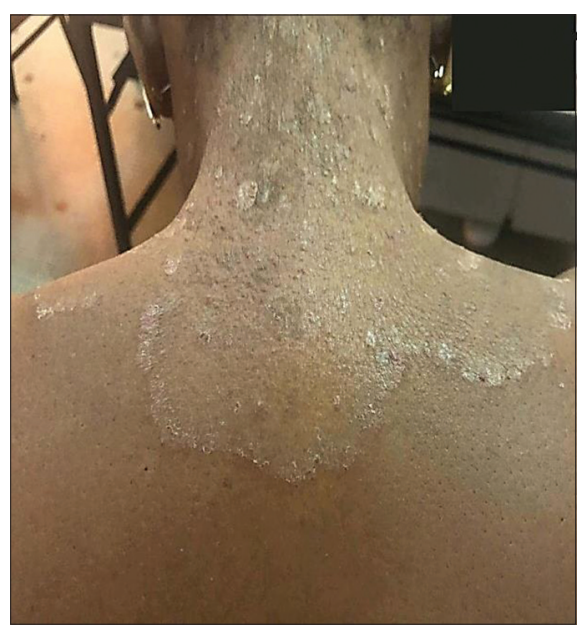

Figure 4: Dermatophytie du cou au cours du lupus systémique. rapportait que la dose cumulée était un facteur de risque principal des infections. Le taux d'infection serait deux fois plus élevé chez des patients recevant 1 à $20 \mathrm{mg}$ de prednisone et quatre fois plus élevé chez les patients recevant $50 \mathrm{mg}$ par jour [3]. Dans notre étude, le lien n'était pas statistiquement significatif entre la dose de corticoïdes et le nombre d'épisodes infectieux. Le faible risque infectieux retrouvé chez nos patients sous forte dose de corticoïdes $(29,8 \%)$ pourrait être expliqué par le suivi rapproché et le bilan infectieux pré-thérapeutique exhaustif réalisé avant de les traiter par des corticoïdes. La majorité des patients (70.2\%) avaient développé des infections aux doses de corticoïdes inférieures à $20 \mathrm{mg}$ par jour. La durée prolongée de la corticothérapie semble être un facteur inductif. Les infections fongiques étaient plus fréquentes au cours du lupus systémique (55\%) et au cours des connectivites mixtes (56\%). Il s'agissait de mycoses superficielles à type de dermatophyties

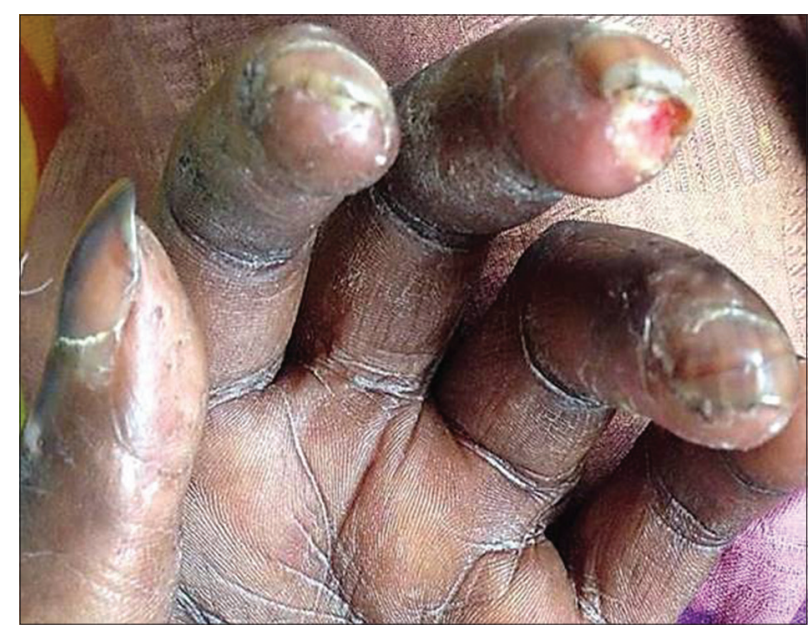

Figure 5: Dactylite au cours du syndrome de Raynaud dans la sclérodermie systémique.

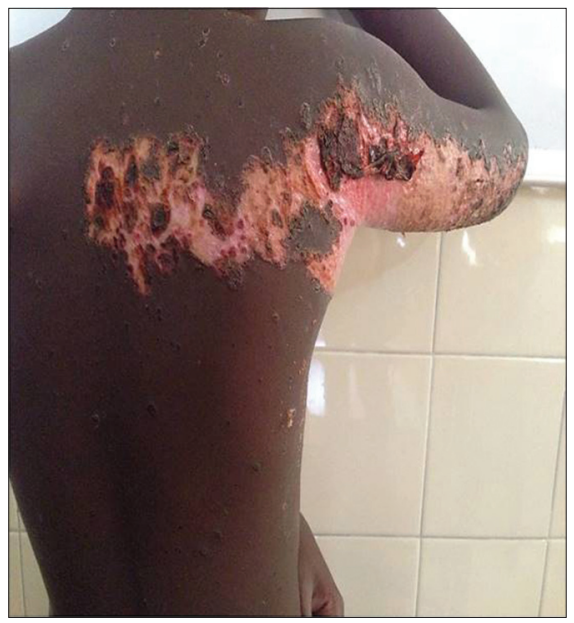

Figure 6: Zona intercostal et brachial au cours d'une connectivite mixte (lupus et dermatomyosite). 
Table 2: Répartition des malades selon le type d'infection

\begin{tabular}{|c|c|c|c|c|}
\hline Type infection & sclérodermie & Lupus & Dermatomyosite & Connectivite mixte \\
\hline \multirow[t]{2}{*}{ Virale } & Herpès cutané & Herpes cutané & Herpès cutané & Zona intercostal \\
\hline & & Zona intercostal & Zona brachial & Herpès génital \\
\hline \multirow[t]{6}{*}{ Bactérienne } & Dactylite & Otite moyenne & Erysipèle de jambe & Cystite \\
\hline & Pneumopathie & Pneumopathie & Pneumopathie & Carie dentaire \\
\hline & Gastroentérite & Tuberculose & Abcès parties molles & Impétigo \\
\hline & Cystite & & Impétigo & Gastroentérite \\
\hline & Carie dentaire & & & Dactylite \\
\hline & Impétigo & & & \\
\hline \multirow[t]{4}{*}{ Mycosique } & Candidose buccale & Dermatophytie cutanée & Candidose cutanée & Dermatophytie cutanée \\
\hline & Dermatophytie cutanée & Teigne cuir chevelu & $\begin{array}{l}\text { Dermatophytie } \\
\text { cutanée }\end{array}$ & Teigne cuir chevelu \\
\hline & Pytiriasis versicolor & Candidose & & Candidose buccale \\
\hline & Teigne cuir chevelu & & & Pytiriasis versicolor \\
\hline \multirow[t]{2}{*}{ Parasitaire } & Gale & Gale & Gale & $\begin{array}{l}\text { Vaginose à Trichomonas } \\
\text { vaginalis }\end{array}$ \\
\hline & & Vaginose à Trichomonas vaginalis & & \\
\hline
\end{tabular}

Table 3: Les traitements locaux et généraux des infections

\begin{tabular}{llcc}
\hline $\begin{array}{l}\text { Classe } \\
\text { thérapeutique }\end{array}$ & Molécule & $\begin{array}{c}\text { Traitement } \\
\text { général } \\
\text { (nombre) }\end{array}$ & $\begin{array}{c}\text { Traitement } \\
\text { local } \\
\text { (nombre) }\end{array}$ \\
\hline \multirow{3}{*}{ Antifongiques } & Fluconazole & 7 & \\
& Terbinafine & 2 & 3 \\
& Griséofulvine & 14 & 4 \\
& Kétoconazole & & 4 \\
& Cyclopiroxolamine & & 17 \\
Antibiotiques & Amoxicilline & 17 & \\
& Ceftriaxone & 5 & \\
& Doxycycline & 5 & \\
& Métronidazole & 2 & \\
& Ciprofloxacine & 5 & \\
& Spiramycine & 2 & \\
& Acide fucidique & & \\
Antiviraux & RZHE & 1 & \\
Antiparasitaires & Acyclovir & 8 & \\
& Benzoate de & & \\
\hline RHZE * : R (rifampicine), H (isoniazide), Z (pyrazinamide), E (ethambutol)
\end{tabular}

cutanées (fig. 4) et les candidoses buccales et génitales, ceci étaient concordant avec l'étude de Diallo et al [16] qui a montré qu'elles étaient les plus fréquentes et parfois une des circonstances de découverte du lupus systémique dans $33 \%$.

Pour les infections bactériennes, elles étaient majoritaires dans plus de la moitié des cas $(55,2 \%)$ au cours de la sclérodermie systémique, il existait un lien statistiquement significatif $(p=0,048)$. Les dactylites (fig. 5) étaient favorisées par le phénomène de Raynaud présent chez les 5 malades ayant développés cette complication ainsi que les ulcères digitaux qui pouvaient être d'origine vasculaire.

La gastroentérite infectieuse était secondaire au syndrome de malabsorption et à la pullulation microbienne intestinale observée chez 10 à $25 \%$ des malades atteints de sclérodermie [17-19]. Nous avons noté un seul cas de tuberculose multifocale au cours du lupus systémique avant le traitement par les corticoïdes. Elle constituait la circonstance de découverte du lupus.

Les infections virales étaient fréquentes au cours de la dermatomyosite avec un lien statistiquement significatif $(\mathrm{p}=0,018)$. Il s'agissait d'infections cutanées à Herpès simplex (fig. 6) et à Virus Varicelle-zona concordant avec les études antérieures qui ont observé une haute prévalence de ces deux virus [20-22]. Les infections parasitaires étaient les moins fréquentes elles étaient représentées par la vaginose à Trichomonas vaginalis et la gale.

Lévolution était favorable dans $87,5 \%$ avec un traitement anti infectieux spécifique. Nous n'avons noté aucun cas de décès secondaire aux infections. Les facteurs de mauvais pronostic rapportées dans la littérature étaient les infections opportunistes telles 
que les pneumopathies à Pneumocystis carinii, les candidoses profondes, les aspergilloses pulmonaires et les pneumopathies à cytomégalovirus [23].

\section{CONCLUSION}

Les connectivites constituent un groupe de maladies auto-immunes dont la prise en charge repose essentiellement sur la corticothérapie et ou les immunosuppresseurs. Limmunodépression secondaire à ces maladies de système et la corticothérapie constituent un risque infectieux majeur au cours de leur prise en charge. La recherche de ces infections au début et au cours du traitement de ces maladies de système permet d'améliorer le pronostic des malades et leurs suivis au long court.

\section{RÉFÉRENCES}

1. Bussone G, Berezné A, Mouthon L. Complications infectieuses de la sclérodermie Systémique. Presse Med. 2009;38:291-302.

2. Godeau B, Mortier E, Roy PM, Chevret S,Bouachour G, Schlemmer B et al. Short and long term outcome about patients with systemic rheumatic diseases in intensive care units: a prognostic study of 181 patients. J Rheumatol. 1997; 24:1317-23.

3. Noel V, Lortholary O, Casassus P, Cohen P, Généreau T, André MH et al. Risk factors and prognostic influence of infection in a single cohort of 87 adults with systemic lupus erythematosus. Ann Rheum dis. 2001;60:1141-4.

4. Jacinto M, Silva E, Riso N, Moraes-Fontes MF. Determinant Factors of Morbidity in Patients with Systemic Lupus Erythematosus. Acta Med Port. 2017;30:368-72.

5. Cervera R, Khamashta MA, Hughes GR. The Euro-lupus project: epidemiology of systemic lupus erythematosus in Europe. Lupus. 2009;18:869-74.

6. Mouthon L, Mestre C, Poiraudeau S, Marchand C, Guillevin L, Launay O. Low influenza-vaccination rate among systemic sclerosis patients. Rheumatology (Oxford). 2010;49:600-6.

7. Moss KE, Ioannou Y, Sultan SM, Haq I, Isenberg DA. Outcome of a cohort of 300 patients with systemic lupus erythematosus attending a dedicated clinic for over two decades. Ann Rheum Dis. 2002;61:409-13.

8. Stuck AE, Minder CE, Frey FJ. Risk of infection complications in patients taking glucocorticosteroides. Rev Infect Dis. 1989;11:954-74.

9. Shyam C, Malaviya AN. Infection-related morbidity in systemic lupus erythematosus: a clinico - epidemiological study from northern India. Rheumatol Int. 1996;16:1-3.

10. Gladman DD, Hussain F, Ibanez D, Urowitz MB. The nature and outcome of infection in systemic lupus erythematosus. Lupus. 2002;11:234-9.

11. Steen VD, Medsger TA. Changes in causes of death in systemic sclerosis, 1972-2002. Ann Rheum Dis. 2007;66:940-4.

12. Zonana-Nacach A, Camargo-Coronel A, Yanez P, Sánchez L, Jimenez-Balderas FJ, Fraga A. Infections in outpatients with systemic lupus erythematosus: a prospective study. Lupus. 2001;10:505-10.

13. Abdessemed A, Khaldoun N, Brahimi N, Aicha Ladjouze-Rezig. Infections au cours des maladies systémiques: sclérodermie systémique et lupus érythémateux disséminé. Rev Mar Rhum. 2015;31:49-59.

14. Barri J, Fessler MD. Infectious diseases in systemic lupus erythematosus: risk factors, management and prophylaxis. Best Pract Res Clin Rheumatol. 2002;16:281-91.

15. Bosch X, Guillabert A, Pallarès L, Cerveral R, RamosCasals M,Bové A, et al. Infections in systemic lupus erythematosus: a prospective and controlled study of 110 patients. Lupus. 2006;15:528-34.

16. Diallo M, Diatta BA, Diop A, Ndiaye MT, Ndiaye M, Seck B et al. Lupus erythematosus in Senegal: study of 340 Cases. Dermatol Case Rep. 2017;2:3.

17. Gough A, Andrews D, Bacon PA, Emerit P. Evidence of omeprazole-induced small bowel bacterial overgrowth in patients with scleroderma. Br J Rheumatol. 1995;34:976-7.

18. Domenech E, Kelly J. Swallowing disorders. Med Clin North Am. 1999;83:97-113.

19. Domsic R, Fasanella K, Bielefeldt K. Gastrointestinal manifestations of systemic sclerosis. Dig Dis Sci. 2008;53:1163-74.

20. Jallouli M, Frigui M, Marzouk S, Mâaloul I, Kaddour N, Bahloul Z. Complications infectieuses au cours du lupus érythémateux systémique: étude de 146 patients. Rev Med Interne. 2008;29:626-31.

21. Hashimoto A, Okuyama R, Watanabe H, Tagami H, Aiba S. Cytomegalovirus infection complicating immunosuppressive therapy for dermatomyositis. Acta Derm Venereol. 2006;86:535-7.

22. Nived O, Sturfelt G, Wollheim F. Systemic lupus erythematosus and infection: a controlled and prospective study including an epidemiological group. QJmed.1985;55:271-87.

23. GinzlerE,DiamondH,KaplanD, WeinerM,SchlesingerM,SeleznickM. Computer analysis of factors influencing frequency of infection in systemic lupus erythematosus. Arthrit Rheum. 1978;21:37-44.

Copyright by Boubacar Ahy Diatta, et al. This is an open access article distributed under the terms of the Creative Commons Attribution License, which permits unrestricted use, distribution, and reproduction in any medium, provided the original author and source are credited. Source of Support: Nil, Conflict of Interest: None declared. 